\title{
A Vesicle-Coated Particle Method for the Determination of Electrophoretic Mobility of Gastric Vesicles
}

\author{
Noriaki Takeguchi*, Tadashi Kashiwagura and Magotoshi Morii \\ Faculty of Pharmaceutical Sciences, Toyama Medical and Pharmaceutical \\ University, Toyama, 930-01, Japan
}

\begin{abstract}
In the presence of both ATP and $\mathrm{Mg}^{++}$, the density of the negative charge on the external surface of hog gastric vesicles had a higher (absolute) value in comparison to values in the presence of ATP only or the absence of both ATP and $\mathrm{Mg}^{++}$as seen from the electrophoretic mobilities measured with a conventional cytopherometer. Gastric vesicle-coated particles of n-alkanes or n-alkyl halides were used for the study, in which their surfaces were examined under differential interference-contrast microscopy. This showed that the surfaces were coated by the vesicles. On the addition of Mg-ATP, an increase in the density of the surface charge took place irrespective of the oil used. With $\mathrm{n}$-alkyl bromide $(<0.1 \% \mathrm{v} / \mathrm{v})$, reorganization of the vesicular structure was indicated from the measurements of proton uptake by the vesicles. This uptake was followed by an increase in the proton leakiness of the membrane. This is evidence that the increased density of the negative charge of the vesicle surface is not due to the proton gradient but with most to phosphorylation of the $\mathrm{K}^{+}$-ATPase in the gastric vesicles. The temperature dependence of the viscosity-corrected electrophoretic mobility showed that there was some transition about $23^{\circ} \mathrm{C}$ in the presence of $\mathrm{Mg}$-ATP, but not in the absence of Mg-ATP.
\end{abstract}

Purified gastric vesicles derived from acid-secreting parietal cells of the gastric epithelium contain a $\mathrm{K}^{+}$-activated ATPase (M.W. ca. 100,000) as a major protein $(2,6,12,14)$. Due to the ATPase, these vesicles can simultaneously translocate $\mathrm{H}^{+}$ and $\mathrm{K}^{+}$, apparently by an electroneutral exchange, to produce a high intravesicular concentration of $\mathrm{H}^{+}$. Based on measurements of free-flow electrophoresis, Saccomani et al. reported that there is an increase in the electrophoretic mobility of the vesicle in the presence of Mg-ATP which they attributed to enzyme phosphorylation (13).

Because there is increasing concern about the negative surface charges of vesicles, we have made a study of the electrophoretic mobility of the vesicle with a cytopherometer (its microscope magnification; $800 \times$ ). However, the diameter of the gastric vesicles (ca. $0.2 \mu \mathrm{m}$ ) was not great enough to measure with the cytopherometer. Therefore, following a classical procedure, a small amount of n-alkane (or its halide derivative) which exceeded the aqueous solubility was mixed with the vesicle

* To whom correspondence should be addressed.

Abbreviations used are: Pipes, 1,4-piperazinediethane sulfonic acid; $\mathrm{K}^{+}$-ATPase, potassium stimulated ATP phosphohydrolase; TCS, tetrachlorosalicylanilide; DIC, differential interferencecontrast microsopy. 
suspension. This produced observable particles of about $0.8-8 \mu \mathrm{m}$. The electrophoretic mobilities of the particles were significantly small in comparison to those of oil droplets of about the same size, and seemed to reflect the density of the charge on the vesicular surface. We studied the effects of ATP and temperature on mobility. Proton uptake by vesicles in the presence of n-alkane or n-alkyl halide also was checked.

\section{MATERIALS AND METHODS}

Gastric vesicles. The gastric vesicle suspension was obtained as follows. Hog stomachs were placed on ice immediately after the animals had been slaughtered. All the tissue manipulations and fractionations were carried out in a cold room. The epithelium from the fundic portion was scraped with a stainless steel spatula. The scrapings were placed in $0.25 \mathrm{M}$ sucrose, then homogenated in a teflon homogenizer by 3-5 passes of its rotating teflon pestle (ca. 2,000 rpm). This homogenate was diluted with $0.25 \mathrm{M}$ sucrose and centrifuged at $2,000 \times \mathrm{g}$ for $1 \mathrm{~h}$. The supernatant was recentrifuged at $78,000 \times \mathrm{g}$ for $1 \mathrm{~h}$ to produce the crude microsome pellet. The pellets were resuspended in $0.25 \mathrm{M}$ sucrose and layered over a discontinuous sucrose density gradient consisting of $12 \mathrm{ml}$ of $0.25 \mathrm{M}$ sucrose, $10 \mathrm{ml}$ of $7 \%$ ficoll- $0.25 \mathrm{M}$ sucrose, and $10 \mathrm{ml}$ of $30 \%$ sucrose. After centrifuigng the suspension for $40 \mathrm{~min}$ at $132,000 \times \mathrm{g}$ in a vertical rotor, a vesicle band showed up at the $0.25 \mathrm{M}$ sucrose- $7 \%$ ficoll interface. The preparation had a protein concentration of $2.0-2.3 \mathrm{mg}$ protein $/ \mathrm{ml}$.

Chemicals. Valinomycin was purchased from P-L Biochemicals. N-alkanes and their halide derivatives (carbon number: 6-14) were purchased from Tokyo Kasei. The sodium salt of ATP was from Sigma (vanadate-free). All other chemicals were of reagent grade.

Particles composed of vesicle-oil for electrophoretic measurements. One part of gastric vesicle suspension was diluted 5-fold by adding 4 parts of $\mathrm{KCl}$ medium composed of $150 \mathrm{mM}$ $\mathrm{KCl}, 2 \mathrm{mM} \mathrm{MgCl}$, $2 \mathrm{mM}$ Pipes-tris ( $\mathrm{pH}$ 7.4) unless otherwise noted. A volume of $0.1 \%$ $(\mathrm{v} / \mathrm{v})$ of $\mathrm{n}$-alkane or $\mathrm{n}$-alkyl halide was added to the diluted vesicle suspension, which was agitated with a voltex mixer for $30 \mathrm{sec}$. The suspension then was centrifuged for $1 \mathrm{~h}$ at $1,000 \times \mathrm{g}$, after which its pellet was resuspended in 5 parts $\mathrm{KCl}$ medium. This pellet suspension was used for the measurements of electrophoretic mobility. N-alkane with a relatively short hydrocarbon chain gave a smaller amount of pellet than its corresponding halide derivative. With n-hexyl bromide, the amount of total protein in the pellet fraction was about $19 \%$ of the initial total protein in the vesicle suspension.

Apparatus. A Carl Zeiss cytopherometer was used for the measurements of electrophoretic mobility, usually with a $2-8 \mathrm{~mA}$ current. Experiments were made at $25^{\circ} \mathrm{C}$ unless otherwise stated. All values are averages $\pm \mathrm{SE}$ (no. of observations). Student t-tests were carried out to determine significance. Microscopic examinations of the preparations also gave the approximate sizes of the particles measured. We did not measure large coagulated particles. The distribution of the diameters of the particles was 0.8 to $8 \mu \mathrm{m}$ with most from 1.5 to $3.5 \mu \mathrm{m}$. The specific resistance of the medium was measured with a conductivity cell and a universal bridge.

The electrophoretic mobility of rat erythrocytes was measured in (1/15)M phosphate buffer $(\mathrm{pH} 7.3)$ which included $5.4 \%$ glucose to check the accuracy of the measurements. A value of $-1.132 \pm 0.013(20) \mathrm{cm}^{2} \mathrm{sec}^{-1} \mathrm{~V}^{-1} \times 10^{-4}$ was obtained, which agrees well with the reported value of $-1.100 \pm 0.008$ (ref. 18).

A Nikon S-KeII microcope was used in the differential interference-contrast (Nomarski) microscopy. 
Proton uptake measurements. The gastric vesicle suspension was diluted to $0.2 \mathrm{mg}$ protein/ml in buffer of $150 \mathrm{mM} \mathrm{KCl}, 2 \mathrm{mM} \mathrm{MgCl} 2,5 \mathrm{mM}$ glycylglycine (pH 6.11) and kept at room temperature for $2 \mathrm{~h}$. After the addition of $0.1 \%(\mathrm{v} / \mathrm{v}) \mathrm{n}$-alkane or n-alkyl bromide, the suspension was mixed with a voltex mixer for $60 \mathrm{sec}$. The suspension then was used for the proton uptake experiments without further sedimentation; thus included vesicle-coated particles and free vesicles. A Radiometer $\mathrm{pH}$ meter was used, and the change in the recording potential from the $\mathrm{pH}$ meter was offset partially with an appropriate constant voltage. The out put was amplified by an operational amplifier. Mg-ATP was added to give a final concentration of $0.2 \mathrm{mM}$. Effects of ionophores such as valinomycin (final concentration $10^{-5}$ $\mathrm{g} / \mathrm{ml}$ ) and TCS (a proton ionophore, final concentration $2.5 \mu \mathrm{M}$ ) were studied.

\section{RESULTS}

Electrophoretic mobility of oil droplets. First, the electrophoretic mobility of $\mathrm{n}$-alkane or $\mathrm{n}$-alkyl halide oil droplets was measured. For this purpose, $0.1 \%$ oil was added to $\mathrm{KCl}$ medium and mixed with the voltex mixer for $30 \mathrm{sec}$. Oil droplets seen in the microscopic field were clear and bright. The diameters measured ranged from 0.8 to $8 \mu \mathrm{m}$. Particle size did not have any systematic effect on mobility. The electrophoretic mobility of the oil droplets was about $-4 \mathrm{~cm}^{2} \mathrm{sec}^{-1} \mathrm{~V}^{-1} \times 10^{-4}$ in $\mathrm{KCl}$ medium ( $\mathrm{pH}$ 7.4). For n-tetradecane and n-octyl bromide, the mobilities were $-4.03 \pm 0.08$ (20) and $-4.56 \pm 0.08(20)$, which is in good agreement with known values $(7,16)$. Additions of valinomycin $\left(10^{-5} \mathrm{~g} / \mathrm{ml}\right)$ and $\mathrm{Mg}$-ATP $(3 \mathrm{mM})$ to the medium did not change the mobility of oil droplets significantly. The mobility of n-octyl bromide was $-4.21 \pm 0.06(20) \mathrm{cm}^{2} \mathrm{sec}^{-1} \mathrm{~V}^{-1} \times 10^{-4}$ in the presence of valinomycin and Mg-ATP.

Differential interference-contrast microscopy $(D I C)$ of oil droplets and vesicle-coated particles. DIC photographs of large droplets is shown in Fig. 1a and an extraordinarily large droplet is shown in Fig. 1b. Oil droplets had perfectly circular shapes, without exception. In contrast, gastric vesicle-coated particles had slightly deformed round shapes (in Fig. 1c) when extra-large particles were examined, and their surfaces showed some unevenness. The photograph in Fig. 1d shows the periphery of the particle in sharp focus. Many projections, about 0.5 to $1 \mu \mathrm{m}$ long, protrude from the round surface. These photographs show that gastric vesicles did not cover the oil surface in a single uniform vesicle layer. The projections seem to be composed of oil covered with vesicles. Oil is considered to fill the void spaces among the spherical vesicles that have accumulated on the oil droplet. This would enable the entire surface of an oil droplet to be covered with vesicles which would produce a stable state in term of energy. The smaller sized vesicle-coated particles were so small that the surface structure of the particles could not be seen on the DIC photographs. It must be essentially the same as that of the larger size particles, although the surface projections of the small particles would be less because of the limited amount of core oil. A model showing the profile of a vesicle-coated particle is given in Fig. 2.

The electrophoretic mobility of the gastric vesicle-coated particle. The mobilities of the particles composed of gastric vesicle-oils in $\mathrm{KCl}$ medium at $25^{\circ} \mathrm{C}$ are shown in Table 1; they ranged from about -0.6 to $-0.7 \mathrm{~cm}^{2} \mathrm{sec}^{-1} \mathrm{~V}^{-1} \times 10^{-4}$. There was no significant difference between n-alkane and its halide derivative. The brightness and contrast of these particles under the microscope were rather dull, and differed from the brightness and contrast of the oil droplets, themselves. To increase the contrast, we used a green filter but no other technique was necessary. Together with other 


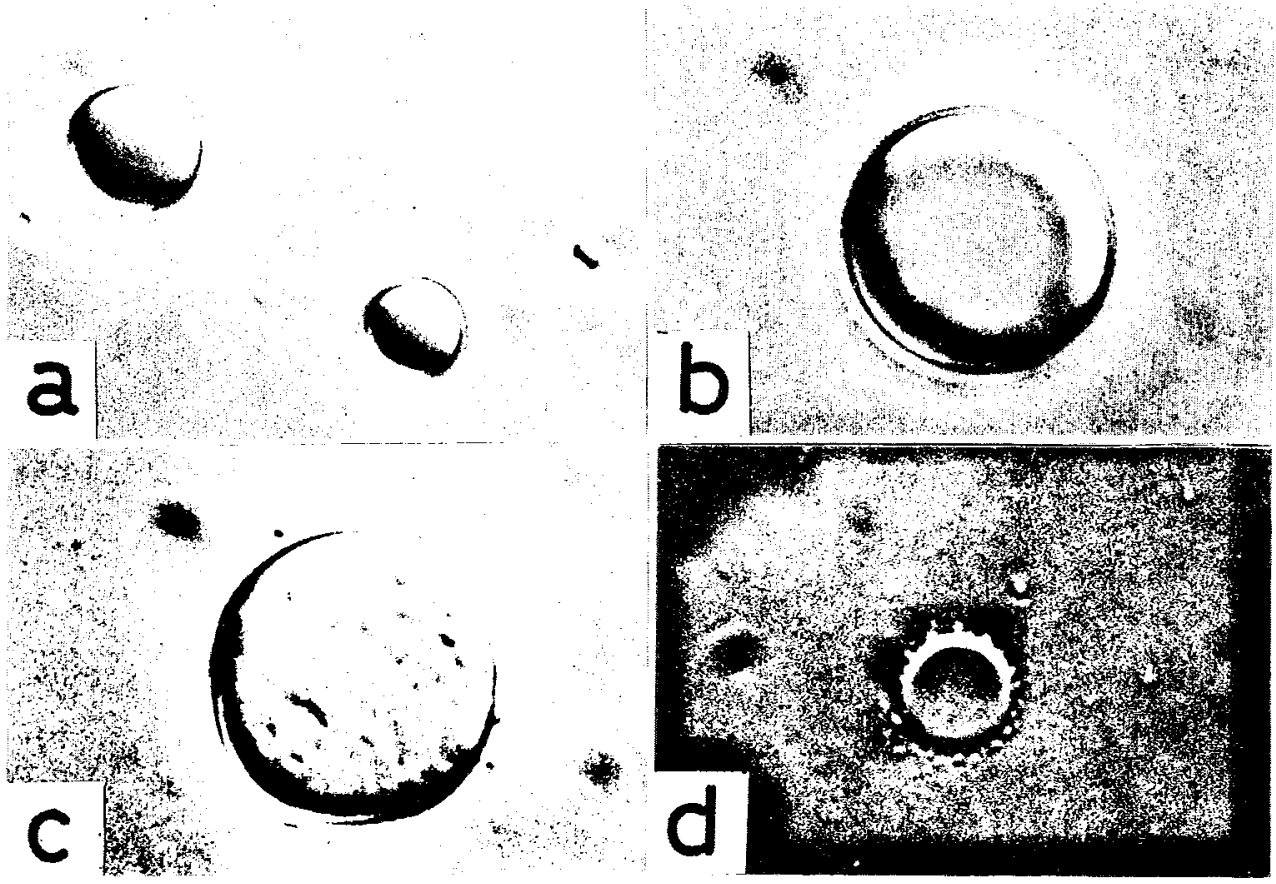

Fig. 1. Differential interference-contrast photographs of oil droplets ( $a$ and $b$ ) and of gastric vesicle-coated particles ( $c$ and $d$ ). The oil is $n$-hexyl bromide. $\times 950$

DIC results, present large decrease (absolute value) in the mobility from about -4 to $-0.6--0.7 \mathrm{~cm}^{2} \mathrm{sec}^{-1} \mathrm{~V}^{-1} \times 10^{-4}$, suggested that the vesicles were adsorbed on the surfaces of the oil droplets.

The addition of valinomycin $\left(10^{-5} \mathrm{~g} / \mathrm{ml}\right)$ and $\mathrm{Mg}-\mathrm{ATP}(3 \mathrm{mM})$ to the $\mathrm{KCl}$ medium significantly increased (absolute value) the electrophoretic mobility (significances listed in Table 1). Because the mobilities of the oil droplets were not affected by the presence of valinomycin and Mg-ATP, this increase in mobility is considered to be

\section{AQUEOUS PHASE}

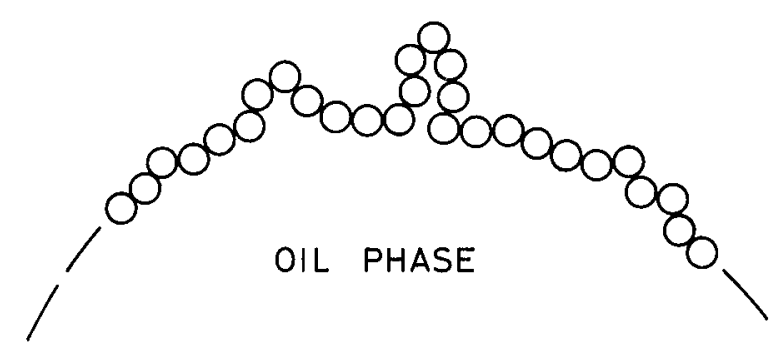

Fig. 2. A model for the surface structure of a vesicle-coated particle (a portion of the profile). Small circles show vesicles. 
TABLE 1. ElECTROPHORETIC MOBILITIES $\left(\mathrm{CM}^{2} \mathrm{SEC}^{-1} \mathrm{~V}^{-1} \times 10^{-4}\right)$ OF PARTICLES COMPOSED OF GASTRIC VESICLE-N-ALKANE OR OF VESICLE-N-ALKYL HALIDE IN KCL MEDIUM AT $25^{\circ} \mathrm{C}$ IN THE ABSENCE (CONTROL) AND THE PRESENCE OF VALINOMYCIN $\left(10^{-5} \mathrm{G} / \mathrm{ML}\right)$ AND MG-ATP $(3 \mathrm{MM})$.

\begin{tabular}{lccc} 
& \multicolumn{1}{c}{ control } & valinomycin and Mg-ATP & significance \\
\hline n-hexane & $-0.627 \pm 0.017(30)$ & $-0.792 \pm 0.017(30)$ & $\mathrm{p}<0.01$ \\
n-hexyl bromide & $-0.634 \pm 0.012(30)$ & $-0.832 \pm 0.016(30)$ & $\mathrm{p}<0.01$ \\
n-heptyl bromide & $-0.711 \pm 0.019(32)$ & $*$ & \\
n-octane & $-0.721 \pm 0.016(30)$ & $-0.837 \pm 0.020(30)$ & $\mathrm{p}<0.01$ \\
n-octyl bromide & $-0.715 \pm 0.084(30)$ & $-0.838 \pm 0.028(30)$ & $\mathrm{p}<0.01$ \\
n-decane & $-0.590 \pm 0.031(25)$ & $*$ & \\
n-decyl chloride & $-0.605 \pm 0.027(30)$ & $-0.720 \pm 0.06(30)$ & $\mathrm{p}<0.05$ \\
n-dodecyl chloride & $-0.708 \pm 0.032(30)$ & $-0.883 \pm 0.022(30)$ & $\mathrm{p}<0.01$ \\
n-tetradecane & $-0.646 \pm 0.018(30)$ & $-0.865 \pm 0.030(30)$ & $\mathrm{p}<0.01$ \\
n-tetradecyl chloride & $-0.705 \pm 0.032(30)$ & $-0.780 \pm 0.022(30)$ & $\mathrm{p}<0.01$ \\
& & $-0.823 \pm 0.023(29) * *$ & $\mathrm{p}<0.01$ \\
\hline
\end{tabular}

* no observations

** observation with Mg-ATP (without valinomycin) for n-tetradecyl chloride.

due to the increase in the density of the surface negative charge on the vesicles adsorbed on the oil droplets. A similar increase was found when only Mg-ATP was added to the medium, which was about equal to that in the presence of both valinomycin and Mg-ATP.

A graphical representation for the particles composed of the vesicle-n-tetradecane is given in Fig. 3.

The increase in the electrophoretic mobility caused by the addition of Mg-ATP probably is due to phosphorylation of the $\mathrm{K}^{+}$-ATPase located in the bilayer structure of vesicle. There also is the possibility that the addition of Mg-ATP induced the partial release of the vesicles from the particle surface, which would cause part of the surface of the oil core to come into direct contact with the aqueous phase. To clarify this point we did the following experiments. The vesicle-coated particles were formed

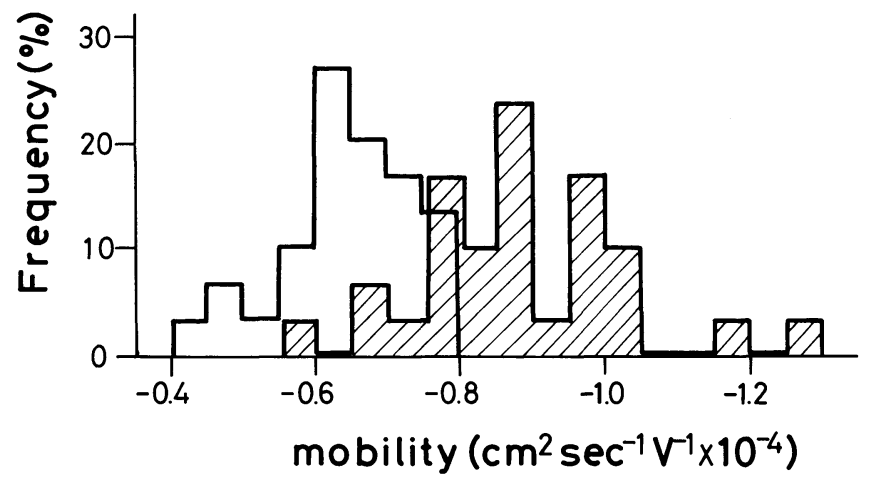

Fig. 3. Distribution of the electrophoretic mobility of particles composed of gastric vesicle-ntetradecane in the absence (open) and presence (shaded) of valinomycin $\left(10^{-5} \mathrm{~g} / \mathrm{ml}\right)$ and $\mathrm{Mg}$-ATP (3 mM). 
TABLE 2. ELECTROPHORETIC MOBILITIES OF GASTRIC VESICLE-COATED PARTICLES (CORE OIL, N-HEXYL BROMIDE) IN DIFFERENT MEDIA. MEDIA WERE BUFFERED WITH 2 MM PIPES-TRIS (PH 7.4).

\begin{tabular}{lc}
\hline \multicolumn{1}{c}{ medium composition } & mobilities in $\mathrm{cm}^{2} \mathrm{sec}^{-1} \mathrm{~V}^{-1} \times 10^{-4}$ \\
\hline $150 \mathrm{mM} \mathrm{KCl}$ & $-0.618 \pm 0.008(30)$ \\
$150 \mathrm{mM} \mathrm{KCl}+1 \mathrm{mM} \mathrm{ATP}$ & $-0.640 \pm 0.006(30)$ \\
$150 \mathrm{mM} \mathrm{KCl}+1 \mathrm{mM} \mathrm{Mg}-\mathrm{ATP}+2 \mathrm{mM} \mathrm{MgCl}_{2}$ & $-0.818 \pm 0.006(29)$ \\
\hline
\end{tabular}

in $\mathrm{Mg}^{++}$-deficient $\mathrm{KCl}$ medium; composed of $150 \mathrm{mM} \mathrm{KCl}$ and $2 \mathrm{mM}$ Pipes-tris ( $\mathrm{pH}$ 7.4)(n-hexyl bromide was oil used). The addition of ATP (without $\mathrm{Mg}^{++}$) to the particle suspension induced no significant change in mobility (Table 2). From recent $\mathrm{K}^{+}$-ATPase studies (17), the enzyme is known to bind with ATP molecules in the absence of $\mathrm{Mg}^{++}$, but phosphorylation does not proceed without $\mathrm{Mg}^{++}$. Our present findings clearly show that the increase in electrophoretic mobility caused by the addition of Mg-ATP is due to enzyme phosphorylation.

Temperature effects on the mobility. For the comparison of mobilities at different temperatures, we obtained the viscosity correction factor $\left(\eta_{t} / \eta_{25}\right)$ by dividing the viscosity value at temperature $t^{\circ} \mathrm{C}\left(\eta_{t}\right)$ by the value for water at $25^{\circ} \mathrm{C}\left(\eta_{25}\right)$. The mobility observed at each given temperature was multiplied by this correction factor; its product is termed the viscosity-corrected mobility. Corrected mobilities for quartz particles and human red blood cells were independent of the temperature (11). Fig. 4a shows the temperature dependence of the viscosity-corrected mobilities of particles composed of vesicle-n-hexane in $\mathrm{KCl}$ medium. The control values showed a very slow increase with increasing temperature. Corresponding values in the

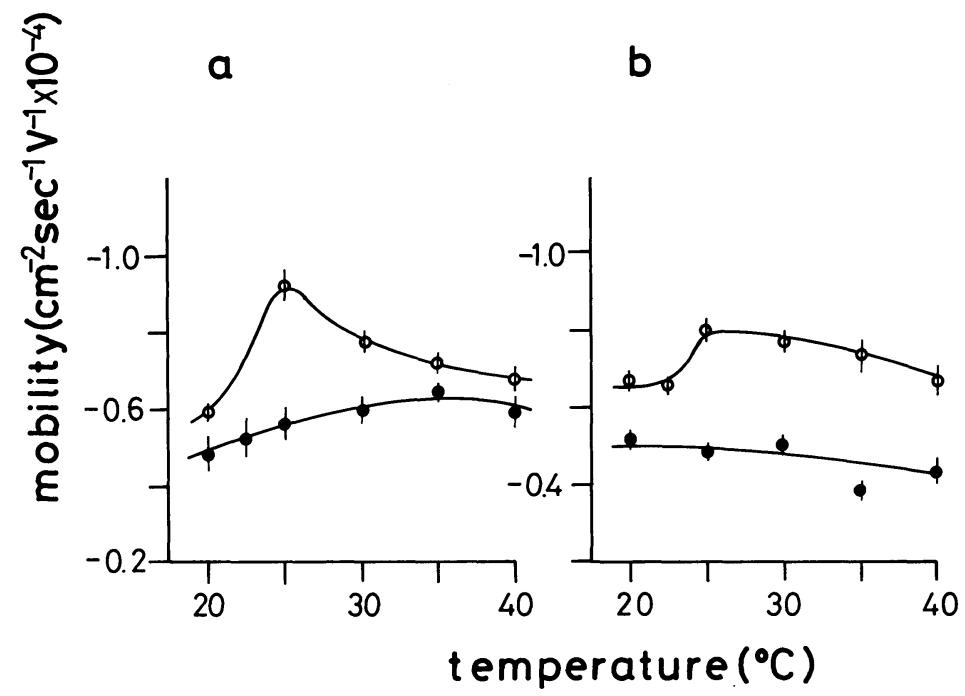

Fig. 4. Viscosity-corrected electrophoretic mobilities of particles composed of gastric vesicle$\mathrm{n}$-hexane (a) and of gastric vesicle-n-hexyl bromide (b). Values are means $\pm \mathrm{SE}$ (20 observations) as a function of temperature. control _$\operatorname{ATP}(3 \mathrm{mM})-\mathrm{O}-$. 

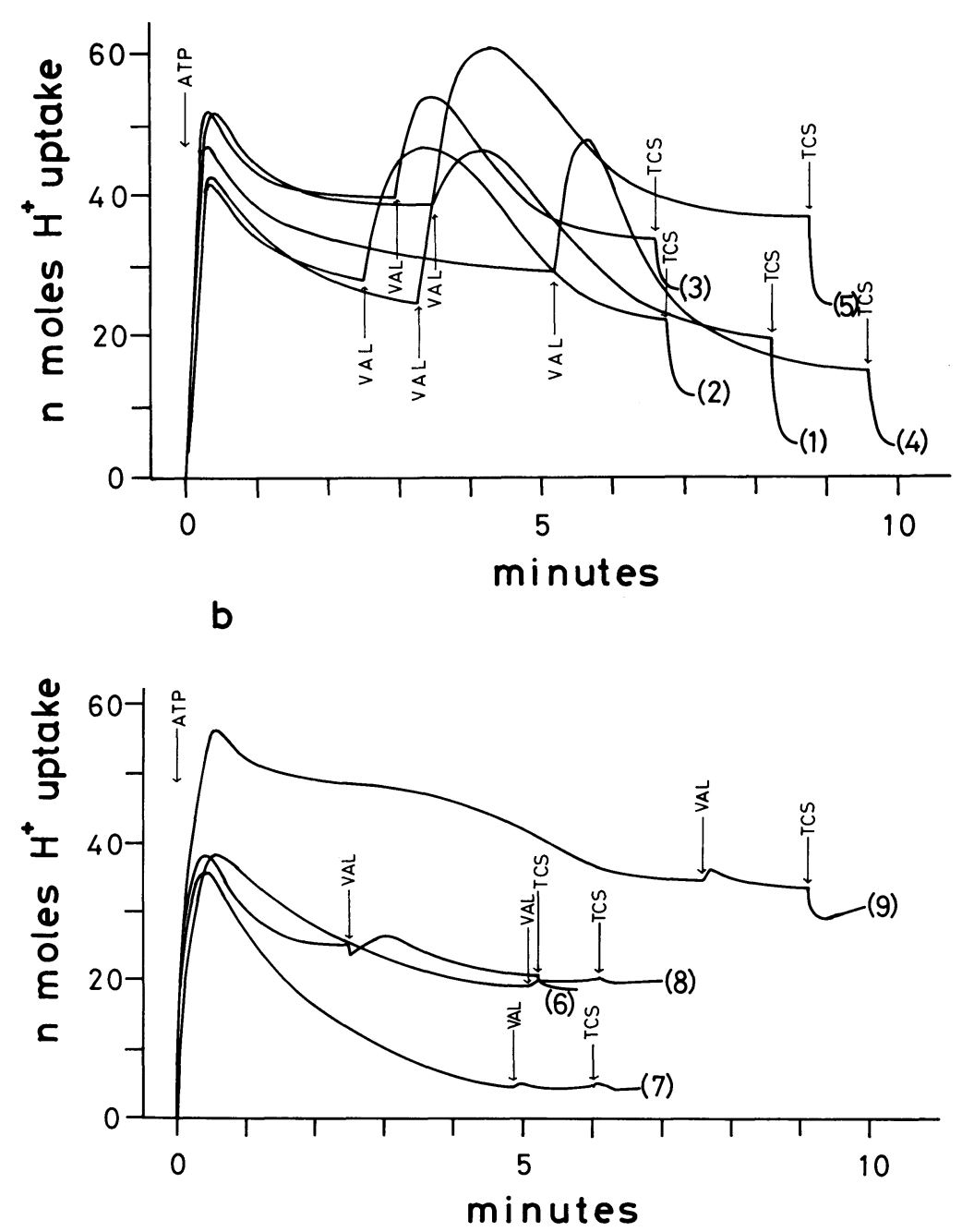

Fig. 5. Proton uptake by gastric vesicles: At zero time, Mg-ATP was added $(0.2 \mathrm{mM})$. At the times indicated, valinomycin $\left(10^{-5} \mathrm{~g} / \mathrm{ml}\right)$ (Val) and TCS $(2.5 \mu \mathrm{M})$ were added. $0.1 \% \mathrm{n}$-alkane (a) or n-alkyl bromide (b) had been added previously to the vesicle suspension. (1) control, (2) n-hexane, (3) n-octane, (4) n-decane, (5) n-tetradecane, (6) n-amyl bromide, (7) n-hexyl bromide, (8) n-heptyl bromide, (9) n-octyl bromide.

presence of Mg-ATP, however, showed a peak around $25^{\circ} \mathrm{C}$. The values for vesiclen-hexyl bromide are shown in Fig. 4b. In the presence of Mg-ATP, mobility showed a transition around $23^{\circ} \mathrm{C}$. Above the transition temperature mobility decreased slowly with increasing temperature as it did for vesicle-n-hexane. In both cases, the mobilities were larger in the presence of Mg-ATP than in its absence.

Proton uptake by gastric vesicles. The presence of $0.1 \%$ n-alkanes did not have a significant effect on proton uptake (Fig. 5a). The addition of Mg-ATP initiated 
proton uptake with an overshoot; this was followed by a slow proton leak. The addition of valinomycin induced a secondary proton uptake due to increased $\mathrm{K}^{+}$ availability $(5,12)$. The addition of TCS in the presence of valinomycin dissipated the proton gradient.

The effects of $0.1 \% \mathrm{n}$-alkyl halides on proton uptake are shown in Fig. 5b. Although the addition of Mg-ATP initiated proton uptake, the proton gradient rapidly selfdissipated, within 5-10 min. Apparently, valinomycin produced no secondary stimulation of proton uptake and the TCS effect indicated that there was no proton gradient left. These probably are the results of the increase in the proton leakiness of the vesicle membrane due to the presence of n-alkyl bromides.

\section{DISCUSSION}

We have demonstrated that the electrophoretic mobilities of particles composed of vesicle-n-alkane or vesicle-n-alkyl halide were very low as compared to the mobility of oil droplets themselves and that the addition of Mg-ATP to the particle suspension increased mobility, whereas the addition of ATP did not increase it in the absence of $\mathrm{Mg}^{++}$.

Electrophoretic mobility reflects the outermost density of the surface charge of a particle; it does not depend on the properites and size of the internal (core) material (3). The large mobility values for oil droplets have been explained as being due to the adsorption of negative ions from the aqueous phase $(3,7,16)$. The electrophoretic mobility of vesicle-coated particles was very small (almost one seventh) as compared to that of the oil droplet itself. The brightness and the contrast of the particles under the microscope also differed from the brightness and contrast of oil droplets. DIC photographs gave additional indication of vesicle coating, and indicated that almost all surface of the particle was coated with vesicles.

Some solubilized oil molecules could penetrate into the bilayer structure of the vesicles and induce some structural change. In the case of a vesicle-coated particle, part of the vesicles surface is in contact with the oil phase, which could induce greater modification in the organization of the vesicular structure. Recent studies of pure phosphatidyl choline liposome, have shown that an excess concentration of short alkanes, such as hexane and octane, decreases the phase transition temperature, and that these agents form an alkane region in the geometric center of the bilayer. Long alkanes, however, such as tetradecane and hexadecane, increase the phase transition temperature and they align parallel to the lipid acyl chains (10). $\mathrm{N}$-alkyl bromides $(1 \%)$ have reported to bring about multiple fusion of the retinal rod outer membrane (8) and to reorganize and fuse artificial phosphatidyl choline liposome (9).

In our experiments, the presence of $0.1 \%$ of an n-alkane did not affect the proton uptake response, whereas an n-alkyl bromide increased the proton leakiness of the vesicle membrane. The amount of the initial proton uptake, however, was the same as that of the control. In the proton uptake experiments, the suspension had two types of vesicles; free vesicles which were not adsorbed on oil droplets (ca. 80\%) and adsorbed vesicles (ca. $20 \%$ ). Therefore, the results of the proton uptake experiments reflected proton uptake and the release behavior in free vesicles. Free vesicles released intravesicularly accumulated protons rapidly in the presence of n-alkyl bromides. Hence, the vesicles adsorbed on the n-alkyl bromides also must leak protons. Since the electrophoretic measurements took about $30 \mathrm{~min}$ for every 30 observations, in- 
cluding the initial set up of 5-10 min (no systematic change with time was observed), the increase in mobility was not due to the proton gradient. This also was true for the n-alkanes, because the proton gradient was small due to leakage over a period of time. Saccomani et al. have reported almost the same amount of increase in electrophoretic mobility (about $0.2 \mathrm{~cm}^{2} \mathrm{sec}^{-1} \mathrm{~V}^{-1} \times 10^{-4}$ ) in the presence of Mg-ATP (13) in experiments on free-flow electrophoresis in $0.25 \mathrm{M}$ sucrose, $8 \mathrm{mM}$ tris acetate buffer at $\mathrm{pH} 7.4$ (no $\mathrm{K}^{+}$, no $\mathrm{Na}^{+}$). Their increase was attributed to phosphorylation of the enzyme because the ATPase can be phosphorylated in the absence of cations such as $\mathrm{K}^{+}$. Our present results, in the presence of $\mathrm{K}^{+}$, coincided with their evidence that a dephosphorylation reaction is the overall rate limiting factor under these conditions (17).

The electrophoretic mobilities of particles composed of vesicle-n-hexane and of vesicle-n-hexyl bromide seemed to have nearly the same transition temperature, about $23^{\circ} \mathrm{C}$. Chang et al. reported the initial proton uptake velocity for gastric vesicles (measured from the $\mathrm{pH}$ change) had a phase transition temperature of $22^{\circ} \mathrm{C}$ with an activation energy of $3.0 \mathrm{kcal} / \mathrm{mol}$ above the transition temperature and $18.1 \mathrm{kcal} / \mathrm{mol}$ below the temperature (1). The good coincidence in the fit of the transition temperature of these two phenomena indicate that the Mg-ATP-induced increase in the density of the surface charge may reflect some conformational change in the enzyme as well as the phosphorylation. Further experimentation is need to confirm this possibility. In similar transport ATPases such as $\left(\mathrm{Na}^{+}+\mathrm{K}^{+}\right)$-ATPase, an alternation in conformation has been reported on phosphorylation (4). Measurements of the free-flow electrophoresis of broken spinach chloroplasts made by Schpendonk et al. showed that the light induced increase in the net surface chaege of chloroplasts is the results of an energy-dependent conformational rearrangement of the components of the thlakoid membrane (15).

Above the transition temperature, the density of the negative charge in the presence of ATP diminished, rather quickly (Fig. 4a) and slowly (Fig. 4b) as the temperature increased. Although there is no clear explanation for this, one possibility is that at high temperatures structural reorganization affects the density of surface charge.

The vesicle-coated particle method can be used with other subcellular substances such as the sarcoplasmic and endoplasmic reticulums. This technique is not affected by the presence of dust (nearly equal in size to the vesicles) because the vesicle-coated particles are large in size as compared with the original vesicular materials.

Acknowledgements. The technical assistance of A. Oka and M. Hiragaki has been greatly appreciated. This work was supported in part by a grant-in-aid for Special Project Research from the Ministry of Education, Science and Culture of Japan.

\section{REFERENCES}

1. Chang, H., G. Saccomani, E. Rabon, R. Schackman and G. Sachs. Proton transport by gastric membrane vesicles. Biochim. Biophys. Acta 464, 313-327, 1977

2. GANSER, A.L. and J.G. ForTE. $\mathrm{K}^{+}$stimulated ATPase in purified microsomes of bullfrog oxyntic cells. Biochim. Biophys. Acta 307, 169-180, 1973

3. Jirgensons, B. and M.E. Straumanis. A short textbook of colloid chemistry. 2nd ed. (translated into Japanese by B. Tamamushi) p. 118, Baifukan, Tokyo, 1962

4. Kuriki, Y., J. Halsey, R. Biltonen and E. RaCker. Calorimetric studies of the interaction of magnesium and phosphate with $\left(\mathrm{Na}^{+}, \mathrm{K}^{+}\right)$ATPase: Evidence of a ligand-induced conformational change in the enzyme. Biochemistry 15, 4956-4961, 1976 
5. Lee, H.C., H. Breitbart, M. Berman and J.G. Forte. Potassium-stimulated ATPase activity and hydrogen transport in gastric microsomal vesicles. Biochim. Biophys. Acta 553, 107-131, 1979

6. Lee, J., G. Simpson and P. Scholes. Change of outer $\mathrm{pH}$ in suspensions of microsomal vesicles accompanying ATP hydrolysis. Biochem. Biophys. Res. Commun. 60, 825-834, 1974

7. Lewis, WM.C.Mc. Größe und Elektrische Ladung der Oelteilchen in Oel-Wasser Emulsionen. Kolloid Z. 4, 211-212, 1909

8. Mason, W.T., S.B. Hladky and D.A. HAydon. Fusion of photorecepter membrane vesicles. J. Membrane Biol. 46, 171-181, 1979

9. Mason, W. and N.G.A. Miller. Fusion of charged and uncharged liposomes by n-alkyl bromides. Biochem. Biophys. Res. Commun. 91, 878-885, 1979

10. McIntosh, T.J., S.A. Simon and R.C. MacDonAld. The organization of n-alkanes in liquid bilayers. Biochim. Biophys. Acta 597, 445-463, 1980

11. Merishi, J.N. and G.V.F. Seaman. Temperature dependence of the electrophoretic mobility of cells and quartz particles. Biochim. Biophys. Acta 112, 154-159, 1966

12. Rabon, E., N. TAKeguchi and G. SAchs. Water and salt permeability of gastric vesicles. $J$. Membrane Biol. 53, 109-117, 1980

13. Saccomani, G., H.B. Stewart, D. Shaw, M. Lewin and G. Sachs. Characterization of gastric mucosal membranes. IX. Fractionation and purification of $\mathrm{K}^{+}$-ATPase-containing vesicles by zonal centrifugation and free-flow electrophoresis technique. Biochim. Biophys. Acta 465, 311330, 1977

14. Sachs, G., H.H. Chang, E. Rabon, R. Schackman, M. Lewin and G. Saccomani. A nonelectrogenic $\mathrm{H}^{+}$pump in plasma membranes of hog stomach. J. Biol. Chem. 251, 7690-7698, 1976

15. Schapendonk, Ad H.C.M., A.M. Merika-Wagner, A.P.R. Theuvenet, H.W. Wong Fong SANG, W.J. Vredenberg and R. KraAyendorf. Energy-dependent changes of the electrophoretic properties of chloroplasts. Biochemistry 19, 1922-1927, 1980

16. SHAw, D.J. Introduction to colloid and surface chemistry (Japanese ed. translated by A. Kitahara and K. Aoki) p. 143, Hirokawa Tokyo, 1970

17. Wallmark, B., H.B. Stewart, E. Rabon, G. Saccomani and G. Sachs. The catalytic cycle of gastric $\left(\mathrm{H}^{+}+\mathrm{K}^{+}\right)$-ATPase. J. Biol. Chem. 255, 5313-5319, 1980

18. Yamada, T. Methods of cytopherometer (in Japanese), p. 45, Bunkodo, Tokyo, 1973 